BULLETIN OF THE

AMERICAN MATHEMATICAL SOCIETY

Volume 81, Number 2, March 1975

\title{
ON THE R-FORMS OF CERTAIN ALGEBRAIC VARIETIES
}

\author{
BY STEPHEN KUDLA
}

Communicated by Dock Rim, October 14, 1974

In this note we determine explicitly the inequivalent models over $\mathbf{R}$ of certain varieties $U=\Gamma \backslash H^{n}$ where $\Gamma$ is a unit group of a totally indefinite quaternion algebra over a totally real number field $k,|k: \mathbf{Q}|=n$, and $H=$ upper half-plane. For each model defined over $\mathbf{R}$ we give a formula for the number of connected components of the manifold of real points of $U$.

1. Let $A$ be a totally indefinite division quaternion algebra over a totally real number field $k$, \& maximal order in $A$, and $\Gamma=\left\{\gamma \in \mathbb{B}^{\times}\right.$with reduced norm $\nu(\gamma)=1$ \}. We fix an isomorphism $\lambda: A_{\mathrm{R}}=A \otimes_{\mathrm{Q}} \mathbf{R} \simeq M_{2}(\mathrm{R})^{n}, n=$ $|k: \mathbf{Q}|$; then $\lambda(\Gamma \otimes 1) \subset \mathrm{SL}_{2}(\mathbf{R})^{n}$ and thus $\Gamma / \pm 1$ acts properly discontinuously on $H^{n}=$ product of $n$ copies of the upper half-plane via fractional linear transformations. Under certain assumptions on $A, \Gamma / \pm 1$ will act without fixed points so that $U=\Gamma \backslash H^{n}$ will be a compact complex manifold. It is well known that such $U$ are imbeddable as nonsingular complex projective algebraic varieties.

A real model of $U$ is a pair $\left(U^{\prime}, \varphi\right)$ consisting of a nonsingular projective variety $U^{\prime} \subset \mathbf{P}^{N}(\mathbf{C})$ defined over $\mathbf{R}$ and a biholomorphic map $\varphi: U \rightarrow U^{\prime}$. Two real models are equivalent if there exists a biregular isomorphism $f$ : $U_{1}^{\prime} \rightarrow U_{2}^{\prime}$ with $f$ defined over $\mathbf{R}$. An equivalence class of real models will be called an R-form of $U$. To each real model $\left(U^{\prime}, \varphi\right)$ of $U$ we associate an antiholomorphic involution $\rho: U \rightarrow U$ by the formula $\rho(x)=\varphi^{-1}(\overline{\varphi(x)})$. We call the points $x \in U$ such that $x=\rho(x)$ the real points of the model $\left(U^{\prime}, \varphi\right)$. The following is well known:

LEMMA 1. The R-forms of $U$ are in one-to-one correspondence with the $\operatorname{Aut}^{h}(U)$ conjugacy classes of antiholomorphic involutions on $U$. Here $\operatorname{Aut}^{h}(U)=$ biholomorphic automorphisms of $U$.

AMS(MOS) subject classifications (1970). Primary 14G05; Secondary 12A60, 22 E40.

Key words and phrases. Real algebraic variety, arithmetic of quaternion algebra, outer automorphisms, antiholomorphic involution. 
2. Now we have an isomorphism $\operatorname{Isom}(U) \simeq \operatorname{Aut}(A, \Gamma) / \Gamma$ where $\operatorname{Aut}(A, \Gamma)=$ subgroup of $\operatorname{Aut}(A)$ preserving $\Gamma$. Notice that if $S=$ the set of primes $p$ of $k$ at which $A_{p}$ is division and $\operatorname{Aut}(k, S)$ is the subgroup of $\operatorname{Aut}(k)$ which perserves the set $S$, then

$$
1 \rightarrow A^{x} / k^{x} \rightarrow \operatorname{Aut}(A) \rightarrow \operatorname{Aut}(k, S) \rightarrow 1
$$

is exact. Certain subsequences of this describe $\operatorname{Isom}(U)$ and $\operatorname{Aut}^{h}(U)$.

THEOREM I. (1) Isom $(U) \supset \operatorname{Aut}^{h}(U)$ are described by

$$
\begin{aligned}
& 1 \rightarrow I(k)_{2} \times Z_{2}^{m} \times U_{k} / U_{k}^{2} \rightarrow \operatorname{Isom}(U) \rightarrow \operatorname{Aut}(k, S, \Gamma) \rightarrow 1, \\
& 1 \rightarrow I(k)_{2}^{\prime} \times Z_{2}^{m^{\prime}} \times U_{k}^{+} / U_{k}^{2} \rightarrow \operatorname{Aut}^{h}(U) \rightarrow \operatorname{Aut}^{h}(k, S, \Gamma) \rightarrow 1
\end{aligned}
$$

where the subgroups are isomorphic to $N_{A} *(\Gamma) / k^{\times} \Gamma$ and $N_{A_{+}}(\Gamma) / k^{\times} \Gamma$ respectively. $I(k)_{2}=$ the two torsion in the ideal class group $I(k)$ of $k, I(k)_{2}^{\prime}=$ image in $I(k)$ of the two torsion of the narrow ideal class group $I_{1}(k), U_{k}$ (resp. $U_{k}^{+}$) = units (resp. totally positive units) of $k$, and the elements of $Z_{2}^{m}$ (resp. $\left.Z_{2}^{m^{\prime}}\right)$ correspond to tuples $\left(e_{1}, \cdots, e_{|S|}\right), e_{i}=0,1$, such that $\left[\Pi_{i=1}^{|S|} p_{i}^{e_{i}}\right] \in I(k)^{2}$ (resp. $\left.\left[\Pi_{i=1}^{|S|} p_{i}^{e_{i}}\right] \in I_{1}(k)^{2}\right), p_{i} \in S$. Finally, there exists a certain 1-cocycle $\tau \rightarrow t_{\tau, \Theta}$ in $Z^{1}\left(\operatorname{Aut}(k, s), I_{1}(k) / I_{1}(k)^{2} I_{1}^{S}(k)\right)$ where $I_{1}^{S}(k)$ is the subgroup of $I_{1}(k)$ generated by the primes of $S$ such that $\operatorname{Aut}^{h}(k, S, \Gamma)=$ $\left\{\tau \in \operatorname{Aut}(k, S)\right.$ such that $\left.t_{\tau, \Theta}=1\right\}$. A similar 1-cocycle with values in $I(k) / I(k)^{2} I^{S}(k)$ describes Aut $(k, S, \Gamma)$.

(2) If $\beta \in N_{A} \times(\Gamma)$ is any element of totally negative reduced norm-such elements exist by strong approximation-then the coset $\beta N_{A} \times(\Gamma) / k^{\times} \Gamma$ in $N_{A} \times(\Gamma) / k^{\times} \Gamma$ consists of all elements of this group which correspond to antiholomorphic involutions in $\operatorname{Isom}(U)$. Finally, since $N_{A^{\times}}(\Gamma) / k^{\times} \Gamma$ is abelian, any two elements $x$ and $x^{\prime}$ in $\beta N_{A} \times(\Gamma) / k^{\times} \Gamma$ correspond to Aut $^{h}(U)$ conjugate involutions $\Longleftrightarrow \exists \tau \in \operatorname{Aut}^{h}(k, S, \Gamma)$ such that $x^{\prime}=x^{\tau}$.

COROllary. If we let $[\beta]$ denote the element of $I(k)_{2} \times Z_{2}^{m} \times$ $U_{k} / U_{k}^{2} \simeq N_{A} \times(\Gamma) / k^{\times} \Gamma$ corresponding to $\beta$, then the set

$$
[\beta] I(k)_{2}^{\prime} \times Z_{2}^{m^{\prime}} \times U_{k}^{+} / U_{k}^{2} / \operatorname{Aut}^{h}(k, S, \Gamma)
$$

is in one-to-one correspondence with a subset of the R-forms of $U$.

REMARK. The theorem classifies only those R-forms coming from involutions which do not permute the factors of $H^{n}$; of course if $\operatorname{Aut}(k, S)$ is trivial, these are all.

3. We now restrict ourselves to those antiholomorphic involutions and 
R-forms arising from the elements of $N_{A} \times(\Gamma) / k^{\times} \Gamma$. We have the following criterion:

LEMmA 2. An antiholomorphic involution $\rho$ of $U$ has a fixed point $\Longleftrightarrow$ $\exists \beta \in N_{A} \times(\Gamma)$ representing it such that $\beta^{2} \in k^{\times}$. More conveniently, suppose $\alpha \in N_{A} \times(\Gamma)$ is any element representing $\rho$; then $\rho$ has a fixed point on $U \Longleftrightarrow$ the quadratic extension $k(\sqrt{ }-\nu(\alpha))=K$ is imbeddable in $A$.

As for the number of connected components of the real points in the case where $K$ is imbeddable, an easy covering space argument together with a computation analogous to that used by Eichler [1] and Shimizu [3] to compute the trace of Hecke operators yields an explicit formula.

THEOREM II. The number of connected components of the fixed point set of $\rho=2^{s-t-1} h(k)^{-1} \Sigma_{D_{i}} h\left(D_{i}\right)\left|U_{k}: N_{K / k}\left(D_{i}^{\times}\right)\right|$where $s=|S|, t=$ number of primes of $S$ which are ramified in $K / k, h(k)=$ class number of $k, D_{i}$ runs

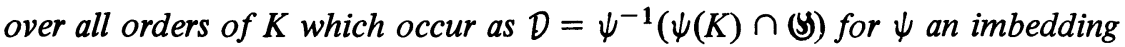
of $K$ into $A$ which satisfies $\psi(\sqrt{ }-\nu(\alpha)) \in N_{A} \times(\Gamma) . h\left(D_{i}\right)=$ class number of the order $D_{i}, D_{i}^{\times}=$units of $D_{i}$, and $N_{K / k}$ is the usual norm from $K$ to $k$.

Notice that the sum is finite because each order of the above sort must contain the order $\left(\mathbb{S}_{K}^{\prime}=\mathbb{S}_{k}+\mathbb{S}_{K} \cap\left(k^{\times} \sqrt{ }-\nu(\alpha)\right), \mathbb{S}_{K}=\right.$ maximal order of $K$.

I have recently discovered that Professor Shimura is working on similar questions.

\section{REFERENCES}

1. M. Eichler, Modular correspondences, Lecture notes, Tata Institute of Fundamental Research, Bombay, India.

2. M. Kneser, Strong approximation, Algebraic groups and discontinuous subgroups, Proc. Sypos. Pure Math., Amer. Math. Soc., Providence, R. I., 1966, pp. 187-196. MR 35 \#4225.

3. H. Shimizu, On zeta functions of quaternion algebras, Ann. of Math. (2) 81 (1965), 166-193. MR 30 \#1998.

DEPARTMENT OF MATHEMATICS, STATE UNIVERSITY OF NEW YORK AT STONY BROOK, STONY BROOK, NEW YORK 11790 